\title{
The minimally invasive spinal deformity surgery algorithm: a reproducible rational framework for decision making in minimally invasive spinal deformity surgery
}

\author{
Praveen V. Mummaneni, M.D., ${ }^{1}$ Christopher I. Shaffrey, M.D., ${ }^{2}$ \\ Lawrence G. Lenke, M.D. ${ }^{3}$ Paul Park, M.D. ${ }^{4}$ Michael Y. Wang, M.D., ${ }^{5}$ \\ Frank La Marca, M.D. ${ }^{4}$ Justin S. Smith, M.D., ${ }^{2}$ Gregory M. Mundis JR., M.D., ${ }^{6}$ \\ David O. OKonkwo, M.D., Ph.D. ${ }^{7}$ Bertrand Moal, M.S., ${ }^{8}$ \\ Richard G. Fessler, M.D., Ph.D., ${ }^{9}$ NeEl Anand, M.D., ${ }^{10}$ Juan S. Uribe, M.D., ${ }^{11}$ \\ Adam S. Kanter, M.D. ${ }^{7}$ Behrooz Akbarnia, M.D., ${ }^{6}$ and KaI-Ming G. Fu, M.D., ${ }^{12}$ \\ on behalf of the Minimally Invasive Surgery Section of the International Spine Study \\ Group
}

${ }^{1}$ Department of Neurosurgery, University of California, San Francisco, California; ${ }^{2}$ Department of Neurosurgery, University of Virginia Health System, Charlottesville, Virginia; ${ }^{3}$ Department of Orthopedics, Washington University in St. Louis, Missouri; ${ }^{4}$ Department of Neurosurgery, University of Michigan, Ann Arbor, Michigan; ${ }^{5}$ Department of Neurosurgery, University of Miami, Florida; ${ }^{6}$ San Diego Center for Spinal Disorders, San Diego, California; ${ }^{7}$ Department of Neurosurgery, University of Pittsburgh, Pennsylvania; ${ }^{8}$ Hospital for Joint Disease, New York, New York; ${ }^{9}$ Department of Neurosurgery, Rush University, Chicago, Illinois; ${ }^{10}$ Cedars Sinai, Los Angeles, California; ${ }^{11}$ Department of Neurosurgery, University of South Florida, Tampa, Florida; and ${ }^{12}$ Department of Neurosurgery, Weill Cornell Medical College, New York, New York

Object. Minimally invasive surgery (MIS) is an alternative to open deformity surgery for the treatment of patients with adult spinal deformity. However, at this time MIS techniques are not as versatile as open deformity techniques, and MIS techniques have been reported to result in suboptimal sagittal plane correction or pseudarthrosis when used for severe deformities. The minimally invasive spinal deformity surgery (MISDEF) algorithm was created to provide a framework for rational decision making for surgeons who are considering MIS versus open spine surgery.

Methods. A team of experienced spinal deformity surgeons developed the MISDEF algorithm that incorporates a patient's preoperative radiographic parameters and leads to one of 3 general plans ranging from MIS direct or indirect decompression to open deformity surgery with osteotomies. The authors surveyed fellowship-trained spine surgeons experienced with spinal deformity surgery to validate the algorithm using a set of 20 cases to establish interobserver reliability. They then resurveyed the same surgeons 2 months later with the same cases presented in a different sequence to establish intraobserver reliability. Responses were collected and tabulated. Fleiss' analysis was performed using MATLAB software.

Results. Over a 3-month period, 11 surgeons completed the surveys. Responses for MISDEF algorithm case review demonstrated an interobserver kappa of 0.58 for the first round of surveys and an interobserver kappa of 0.69 for the second round of surveys, consistent with substantial agreement. In at least 10 cases there was perfect agreement between the reviewing surgeons. The mean intraobserver kappa for the 2 surveys was $0.86 \pm 0.15( \pm$ SD) and ranged from 0.62 to 1 .

Conclusions. The use of the MISDEF algorithm provides consistent and straightforward guidance for surgeons who are considering either an MIS or an open approach for the treatment of patients with adult spinal deformity. The MISDEF algorithm was found to have substantial inter- and intraobserver agreement. Although further studies are needed, the application of this algorithm could provide a platform for surgeons to achieve the desired goals of surgery. (http://thejns.org/doi/abs/10.3171/2014.3.FOCUS1413)

KEY WoRds — adult spinal deformity $\quad$ - minimally invasive surgery $\quad$ scoliosis

\footnotetext{
Abbreviations used in this paper: $\mathrm{LIF}=$ lateral interbody fusion; LL-PI = lumbar lordosis/pelvic incidence; MIS = minimally invasive surgery; MISDEF = minimally invasive spinal deformity surgery; $\mathrm{PT}=$ pelvic tilt; $\mathrm{SVA}=$ sagittal vertebral axis; $\mathrm{TLIF}=$ transforaminal lumbar interbody fusion.
}

$\mathrm{W}$ ITH the aging of the US population, adult degenerative scoliosis is increasing in incidence with significant impact on health and disability.,59-11 Surgical correction of adult degenerative scoliosis has traditionally been performed using open surgical 
approaches. Open spinal deformity correction surgery is associated with a large amount of intraoperative blood loss and significant complication rates. ${ }^{6}$ A multicenter study from the International Spine Study Group reviewing 953 adult spinal deformity patients revealed a major complication rate of $7.6 \%$. The most common complications found in this study were excessive blood loss $(>4$ $\mathrm{L}$ ), deep wound infection requiring reoperation, and pulmonary embolism. ${ }^{12}$

To decrease surgical morbidity and complications, minimally invasive surgery (MIS) approaches for the treatment of adult spinal deformity have recently gained in popularity. However, not every patient with adult spinal deformity is a candidate for MIS. Prior reports have shown that MIS may undercorrect sagittal spinal parameters and result in pseudarthrosis if interbody fusion is not done at every level. , $^{2,16,17}$ Appropriate patient selection for less invasive correction techniques remains controversial.

The main goals for the treatment of adult degenerative spinal deformity are neural element decompression, establishing or maintaining sagittal and coronal global balance, and successfully achieving an arthrodesis. Operative interventions require evaluation of the unique needs and goals of each patient. Several classification schemes as well as levels of treatment have previously been proposed for adult spinal deformity. ${ }^{8}$ In 2010 , Silva and Lenke published a "treatment levels" guide to adult degenerative deformity management. ${ }^{13}$ The authors suggested 6 treatment levels for open spinal deformity surgery, based on clinical and radiographic findings. Mummaneni et al. modified the Lenke-Silva scheme to create an algorithm for minimally invasive treatment of spinal deformity, but this scheme had a high interobserver reliability. ${ }^{8}$ We subsequently created a new algorithm to assist spine surgeons in selecting an appropriate surgical approach for spinal deformity (the minimally invasive spinal deformity surgery [MISDEF] algorithm). This study was designed to evaluate the reliability and consistency of this algorithm in decision making for minimally invasive adult scoliosis.

\section{Methods}

\section{Algorithm Design}

The MISDEF algorithm (Fig. 1) was designed to assist the surgeon in selecting the optimal approach to treating symptomatic adult spinal deformity when considering the use of MIS techniques. This algorithm was created for those patients in whom nonoperative management has failed. The basic parameters for each class are displayed in Table 1. Surgical approaches considered ranged from only MIS techniques to only traditional open techniques. The ultimate selection of the surgical approach was dependent on radiographic parameters as well as symptoms. The initial algorithm comprised 6 potential surgical approaches. ${ }^{8}$ After group discussion and pilot evaluation of case examples, the original algorithm was found to be overly complex with poor interobserver reliability. Consequently, our team of experienced deformity surgeons created a new MISDEF algorithm with 3 general surgical approaches. A Class I approach is MIS or mini-open muscle-sparing decompression alone or MIS fusion of a single listhetic level regardless of curve apex (Fig. 2). The Class I approach is accomplished either through a small tubular port (MIS) or via an expandable tubular port placed through a muscle sparing Wiltse or lateral approach (mini-open). Instrumentation, if placed, may be placed through the expandable port tube or via a percutaneous method. A Class II approach entails MIS or mini-open decompression and interbody fusion of the curve apex or the entire coronal Cobb angle of the major curve (Fig. 3). A Class III approach entails a traditional open surgical approach involving osteotomies and/or extension of fusion to the thoracic spine (Fig. 4). Branch points along the algorithm were based on established ideal sacropelvic parameters and global spinal balance. In general, progressively worse deformity leads to higherclass approaches in the algorithm.

\section{Algorithm Reliability}

The algorithm was provided to 11 fellowship-trained spinal surgeons along with a set of 20 representative cases that were previously published in journals and book chapters. ${ }^{7,8,13,16,17}$ Images included posterior/anterior and lateral scoliosis radiographs as well as select MRI views. A short history of symptoms and the measured radiographic parameters (coronal Cobb angle, pelvic tilt [PT], lumbar lordosis/pelvic incidence [LL-PI] mismatch, and sagittal vertebral axis [SVA]) were provided for each case. Responses were collected and tabulated. After a minimum interval of 2 months, the cases were re-sent to the participating surgeons to be reread. Complete evaluations were obtained for both rounds of assessments.

\section{Statistical Analysis}

Fleiss kappa coefficient analysis was used to determine intraobserver and interobserver reliability. Kappa coefficient values were categorized as 0.00 to 0.20 (slight correlation), 0.21 to 0.40 (fair correlation), 0.41 to 0.60 (moderate correlation), 0.61 to 0.80 (substantial correlation), and 0.81 to 1.00 (almost perfect correlation). All analyses were performed using MATLAB software.

\section{MISDEF Classification}

Class I. Patients with Class I deformity present with symptoms of neurogenic claudication or radiculopathy due to central, lateral recess, or foraminal stenosis and have relatively mild spinal deformity. These patients typically have flexible curves with an LL-PI mismatch of less than $10^{\circ}$, SVA less than $6 \mathrm{~cm}$, a PT less than $25^{\circ}$, minimal if any lateral listhesis $(<6 \mathrm{~mm})$, coronal Cobb angle of less than $20^{\circ}$, and no thoracic hyperkyphosis. These patients tend to have minimal back pain and symptoms with regard to their deformity (Fig. 2). It is important to document preoperatively the absence of Meyerding Grade 2 dynamic instability using flexion and extension radiographs. Patients in this treatment class should have at most an anterior or lateral Meyerding Grade 1 subluxation. The treatment goal in this group of patients is central canal, lateral recess, and/or foraminal nerve root decompression as needed and not correction of their overall mild spinal 


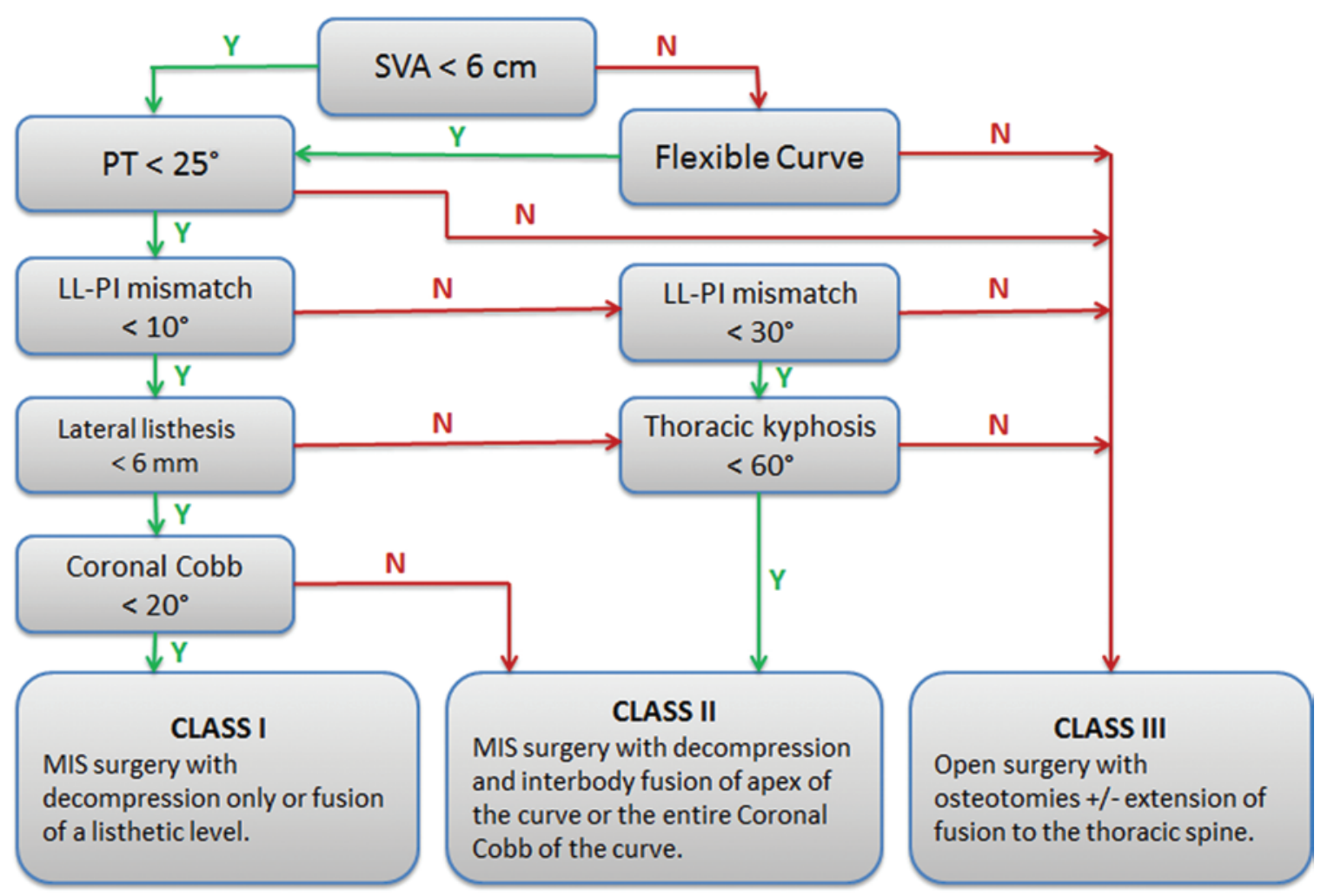

FIG. 1. Minimally invasive spinal deformity algorithm for decision making when considering less invasive correction techniques. $\mathrm{N}=$ no; $\mathrm{Y}=$ yes.

deformity. Minimally invasive techniques are well suited for this type of decompression. Typically, a fixed port tubular retractor or a small expandable tubular retractor is placed via a muscle-sparing approach and is used to perform an ipsilateral hemilaminotomy and foraminotomy. Then, by angling the tubular retractor medially, an undercutting contralateral decompression is also possible ("ipsi-contra" decompression). This type of "ipsi-contra" decompression may be performed at 1 or 2 contiguous levels through one small incision. Radiographically if there is a mobile Grade 1 subluxation at the area of symptomatic stenosis then a 1-level MIS fusion may be added. It is important to understand the context of the limited fusion in light of the given deformity. Ideally the fusion results in parallel endplates in the coronal plane and restoration of lordosis within the segment(s) fused. Fusion can be achieved through an MIS or mini-open transforaminal interbody fusion (TLIF) or mini-open lateral interbody fusion (LIF) and may be supplemented with percutaneous pedicle screw fixation. The surgeon may choose the TLIF or LIF depending on the patient's anatomy (that is, the

TABLE 1: Basic radiographic characteristics of each classification

\begin{tabular}{ccccc}
\hline $\begin{array}{c}\text { MISDEF } \\
\text { Class }\end{array}$ & SVA $(\mathrm{cm})$ & $\mathrm{PT}\left({ }^{\circ}\right)$ & $\begin{array}{c}\text { LL-PI Mismatch } \\
\left({ }^{\circ}\right)\end{array}$ & $\begin{array}{c}\text { Cobb Angle } \\
\left({ }^{\circ}\right)\end{array}$ \\
\hline I & $<6$ & $<25$ & $<10$ & $<20$ \\
II & $>6$ & $<25$ & $<30$ & $>20$ \\
III & $>6$ & $>25$ & $>30$ & $>20$ \\
\hline
\end{tabular}

position of the iliac crest precludes some L4-5 surgeries and L5-S1 is not able to be accessed via LIF).

Class II. Patients in this category have a more dominant back pain component to their chief complaint in addition to their stenosis symptoms (Fig. 3). Candidates for this type of treatment include those who have an LLPI mismatch of $10^{\circ}-30^{\circ}$, a lateral listhesis greater than 6 $\mathrm{mm}$ (Meyerding Grade 2), and/or a coronal Cobb angle of the major curve larger than $20^{\circ}$. Radiographically, these patients typically lack anterior bridging osteophytes and have an SVA of less than $6 \mathrm{~cm}$. Patients who have an SVA of more than $6 \mathrm{~cm}$ but have flexible curves that correct partially when they are supine are also candidates for MISDEF Class II surgery. Minimally invasive techniques are well suited for these cases as they can achieve the same goals as the open approaches, which are typically used for these patients. As with MISDEF Class I, decompression can be achieved at multiple levels through fixed port tubes or expandable tubular retractors. Instrumentation can be performed via percutaneous or mini-open techniques, and interbody grafting is achieved posteriorly via a muscle-splitting approach. In contrast to Class $\mathrm{I}$, more than 1 level of fusion is required, and the fusion extends across the apex of the curve. Additionally, interbody fusion may be accomplished via MIS/mini-open TLIF or LIF across the apex of the curve to obtain some correction of the deformity.

Class III. Patients with Class III disease present with significant coronal and/or sagittal imbalance in addition to back and leg pain (Fig. 4). Patients who need this level of 

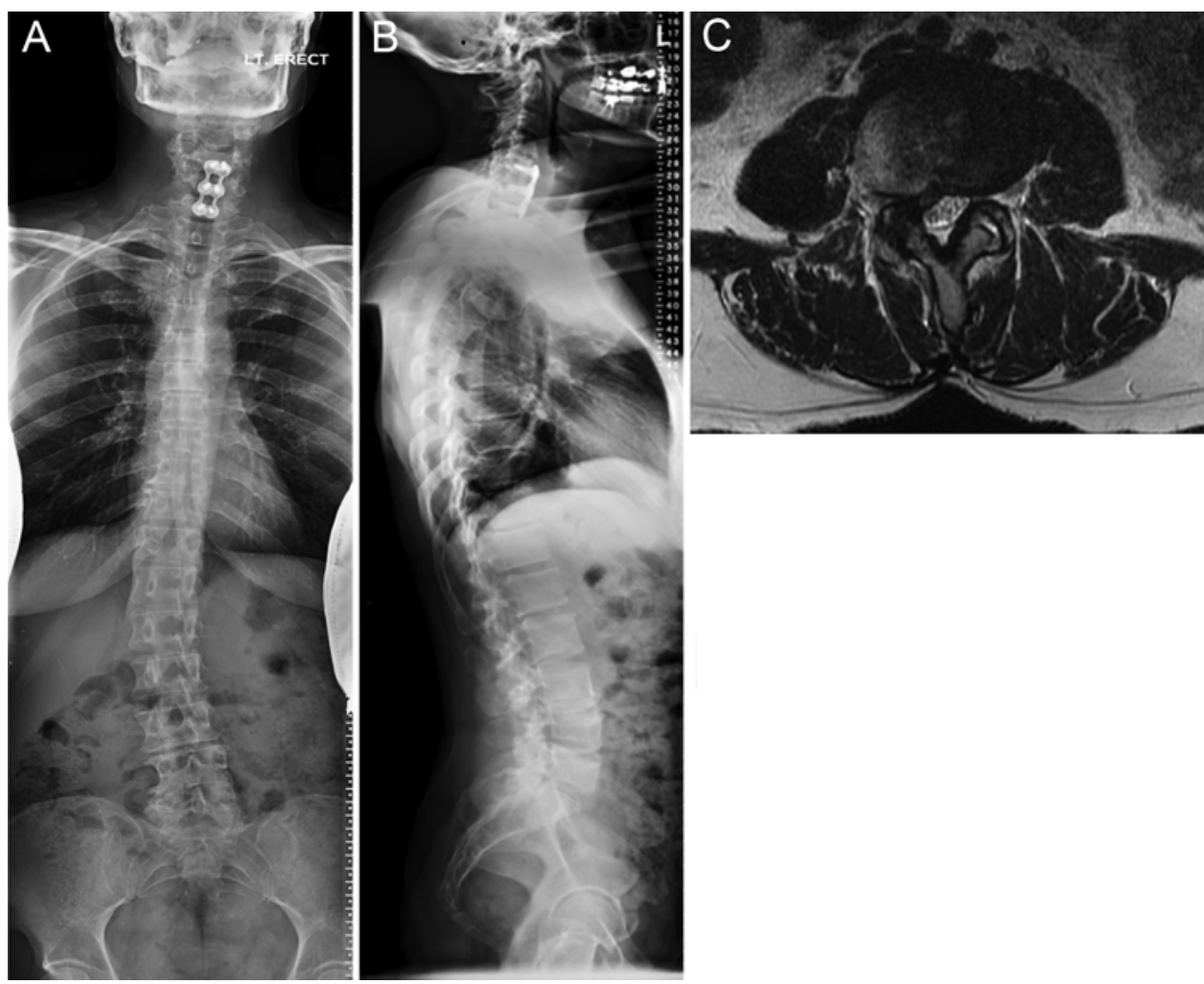

FIG. 2. MISDEF Class I. Anteroposterior (A) and lateral (B) radiographs and axial T2-weighted MR image (C) obtained in a patient with significant leg pain but no back pain. The SVA was less than $6 \mathrm{~cm}$ and the LL-PI mismatch was $7^{\circ}$.

treatment have the following preoperative characteristics: SVA of $7 \mathrm{~cm}$ or greater with a rigid curve, LL-PI mismatch greater than $30^{\circ}$ with a PT greater than $25^{\circ}$, and/or thoracic hyperkyphosis greater than $60^{\circ}$. Patients with Class III disease have a fixed deformity such as iatrogenic flatback deformity frequently requiring 3-column osteotomy for realignment. As these deformities are far more extensive, standard open approaches are preferred, as current minimally invasive techniques do not predictably achieve the intended realignment goals of the surgery.

\section{Results}

Twenty cases of patients of spinal deformity, with preoperative radiographic 36 -in standing scoliosis images and clinical vignettes, were circulated for assessment. Ten cases were consistent with MISDEF Class II, 7 with MISDEF Class III, and 3 with MISDEF Class I; readers could assess the resection type and approach modifier.

Over a 3-month period, 11 surgeons completed the surveys. Table 2 presents the kappa values from the responses. Responses for MISDEF algorithm case review demonstrated an interobserver kappa of 0.58 for the first round of surveys and an interobserver kappa of 0.69 for the second round of surveys, consistent with substantial agreement. In at least 10 cases there was perfect agreement between the reviewing surgeons. The mean intraobserver kappa for the 2 surveys was $0.86 \pm 0.15( \pm$ SD) and ranged from 0.62 to 1 .

\section{Discussion}

Surgery for adult spinal deformity is aimed at alleviation of neurological compression and improvement of spinal balance. Over the past few decades, open surgical techniques such as osteotomies and interbody fusions have been used to effectively treat adult spinal deformity. ${ }^{14,15}$ Over the past decade, minimally invasive techniques have been introduced to reduce the morbidity of spinal surgery, and minimally invasive treatment of adult spinal deformity is now gaining in popularity. However, there are limitations in the degree of deformity correction when utilizing the minimally invasive correction techniques currently available. These techniques are not well suited for the treatment of sagittal imbalance greater than $7 \mathrm{~cm}$, and early reports have shown that these techniques may not restore an ideal SVA of $3.5 \mathrm{~cm}$ in a subset of patients.

We analyzed early reports of minimally invasive spinal deformity treatment and identified preoperative predictors of suboptimal deformity correction. Common preoperative predictors of inadequate correction of spinal balance included rigid curves with an SVA of greater than $6 \mathrm{~cm}$, pelvic tilt greater than $25 \mathrm{~mm}$, LL-PI mismatch greater than $30^{\circ}$, and thoracic hyperkyphosis greater than $60^{\circ} .1,3-5,13,16,17$

In an effort to help guide treatment decision making, we have developed the MISDEF algorithm, which takes these preoperative parameters into account and assists with patient selection. A useful algorithm should 

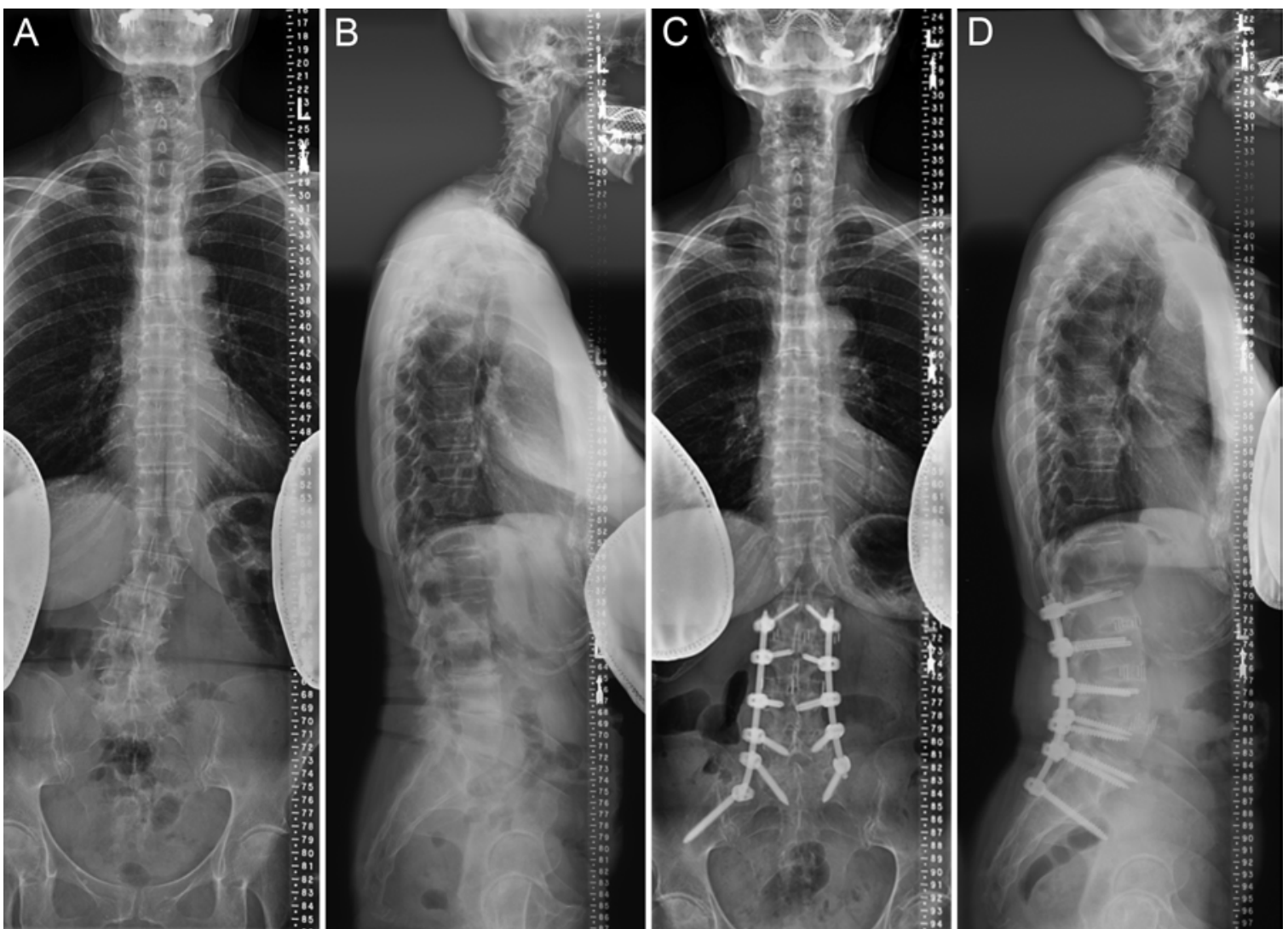

FIG. 3. MISDEF Class II. Preoperative anteroposterior (A) and lateral (B) and postoperative anteroposterior (C) and lateral (D) radiographs obtained in a patient with low-back and leg pain. The SVA was less than $6 \mathrm{~cm}$, and the LL-PI mismatch equaled $13^{\circ}$ preoperatively correcting to $3^{\circ}$ postoperatively.
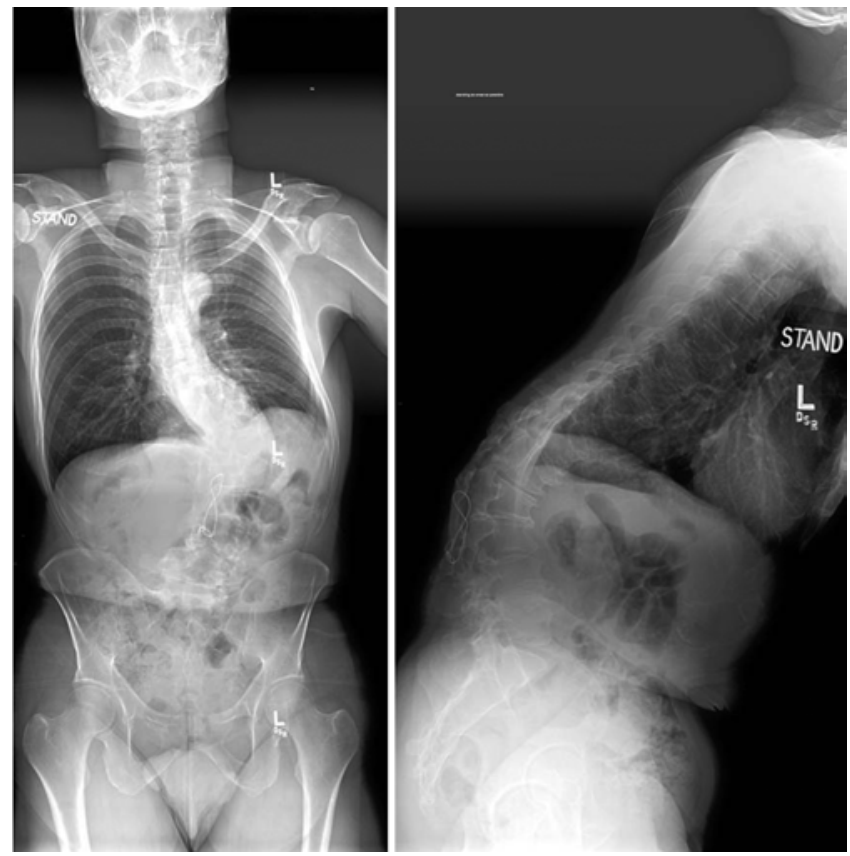

FIG. 4. MISDEF Class III. Anteroposterior (left) and lateral (right) radiographs obtained in a patient with low-back pain and an inability to stand upright. The SVA was greater than $6 \mathrm{~cm}$, the LL-PI mismatch was $25^{\circ}$, and the PT was $25^{\circ}$. ideally be relatively simple to use and should have high interobserver and intraobserver reliability. We created an initial draft of this algorithm that had 6 potential treatment end points and found that the interobserver and intraobserver reliability was low. We revamped the algorithm and simplified it and found that our current version of the MISDEF algorithm has high interobserver and intraobserver reliability.

By using this algorithm, a surgeon can assess if a patient will be a candidate for a 1- or 2-level minimally invasive decompression or limited fusion of a single listhetic level (MISDEF Class I). These patients typically have flexible curves with an LL-PI mismatch of less than $10^{\circ}$, SVA less than $6 \mathrm{~cm}$, PT less than $25^{\circ}$, minimal if any lateral listhesis $(<6 \mathrm{~mm})$, coronal Cobb angle less than $20^{\circ}$, and no thoracic hyperkyphosis.

Patients who have moderate spinal deformity are

TABLE 2: Kappa values for the inter- and intraobserver variability from 2 surveys

\begin{tabular}{cc}
\hline Parameter & Kappa Value \\
\hline intraobserver & 0.86 \\
interobserver & \\
1st round & 0.58 \\
2nd round & 0.69 \\
\hline
\end{tabular}


candidates for MISDEF Class II treatment. This category includes treatment with minimally invasive or mini-open muscle sparing interbody fusion (using tubular or expandable tubular retractors) and pedicle screw fixation to fixate the apex of the coronal curve. Candidates for this type of treatment include those who have an LL-PI mismatch of $10^{\circ}-30^{\circ}$, a lateral listhesis greater than $6 \mathrm{~mm}$, and/or a coronal Cobb angle of the major curve greater than $20^{\circ}$.

Patients with severe deformity are not ideal candidates for minimally invasive spinal surgery given that there is little current evidence that MIS techniques can correct significant deformities, particularly in the sagittal plane. In contrast, open procedures including 3-column osteotomies have been shown to be a reliable method to achieve appropriate correction. Consequently, we suggest that open techniques should be used when treating patients who have the following preoperative characteristics: SVA of $7 \mathrm{~cm}$ or greater with a rigid curve, LL-PI mismatch greater than $30^{\circ}$ with a pelvic tilt greater than $25^{\circ}$, and/or thoracic hyperkyphosis greater than $60^{\circ}$.

This study demonstrates that the MISDEF algorithm is a reproducible and reliable instrument. The results confirm a high intra- and interobserver reliability among a relatively large number of surgeons, which are essential features in a usable algorithm. Although further investigation is now needed to validate the algorithm, the MISDEF provides a tool for assimilating current understanding of adult deformity, as well as MIS techniques and applying it to patient selection for appropriate surgical intervention.

\section{Conclusions}

Careful patient selection and evaluation with the MISDEF algorithm is helpful to select patients with deformity who are appropriate candidates for MIS for spinal deformity. Not all deformity cases can be appropriately treated with minimally invasive techniques. In our opinion, Class III deformities cannot be easily corrected minimally invasively because patients with these deformities often require extensive osteotomies. Our algorithm offers a reliable method for patient selection for deformity surgery using open or MIS approaches. Future studies will be needed to confirm the validity of the MISDEF algorithm in relation to radiographic and health-related quality-of-life outcomes.

\section{Disclosure}

Dr. Mummaneni reports that he receives royalties and honoraria from DePuy Spine, honoraria from Globus, royalties from Thieme publishing, royalties from Quality Medical Publishers, and owns stock in Spinicity. Dr. Shaffrey reports that he is a consultant for Biomet, Globus, Medtronic, NuVasive, and Stryker and is a patent holder with and receives royalties from Biomet and Medtronic. Dr. Lenke reports being a consultant for Medtronic, K2M, and DePuy Synthes Spine; is a patent holder with Medtronic; receives non-study-related clinical or research support from DePuy Synthes Spine, Axial Biotech, AOSpine, SRS, Norton Healthcare, and Fox Family Foundation; and receives royalties from AOSpine North America, Medtronic, and Quality Medical Publishing. Dr. Park reports being a consultant for Globus and Medtronic and receives royalties from Globus. Dr. Wang reports that he is a consultant for and patent holder with DePuy Spine. Dr. La Marca reports that he is a consultant for Globus Medical and Biomet and receives royalties from Globus. Dr. Smith reports that he is a consultant for Biomet, Medtronic, Globus, and DePuy and receives non-study-related clinical or research support from DePuy. Dr. Mundis reports that he is a consultant for NuVasive and $\mathrm{K} 2 \mathrm{M}$; receives non-study-related clinical or research support from NuVasive; and receives fellowship support from Pioneer, NuVasive, and the Orthopaedic Research and Education Foundation. Dr. Okonkwo reports receiving royalties from Lanx. Dr. Uribe reports that he is a consultant for NuVasive. Dr. Kanter reports that he receives non-study-related clinical or research support from NuVasive and royalties from Lanx. Dr. Fu reports that he is a consultant for Medtronic and received an honorarium from DePuy.

Author contributions to the study and manuscript preparation include the following. Conception and design: Fu, Mummaneni, Shaffrey, Lenke, Kanter. Acquisition of data: Fu, Shaffrey, Park, Wang, La Marca, Smith, Mundis, Okonkwo, Fessler, Anand, Uribe, Kanter. Analysis and interpretation of data: Fu, Mummaneni. Drafting the article: Fu, Mummaneni, Park. Critically revising the article: $\mathrm{Fu}$, Mummaneni, Shaffrey, Lenke, Park, Wang, La Marca, Okonkwo, Fessler, Kanter. Reviewed submitted version of manuscript: all authors. Approved the final version of the manuscript on behalf of all authors: Fu. Statistical analysis: Fu, Moal.

\section{References}

1. Acosta FL, Liu J, Slimack N, Moller D, Fessler R, Koski T: Changes in coronal and sagittal plane alignment following minimally invasive direct lateral interbody fusion for the treatment of degenerative lumbar disease in adults: a radiographic study. Clinical article. J Neurosurg Spine 15:92-96, 2011

2. Anand N, Rosemann R, Khalsa B, Baron EM: Mid-term to long-term clinical and functional outcomes of minimally invasive correction and fusion for adults with scoliosis. Neurosurg Focus 28(3):E6, 2010

3. Blondel B, Schwab F, Ungar B, Smith J, Bridwell K, Glassman $S$, et al: Impact of magnitude and percentage of global sagittal plane correction on health-related quality of life at 2-years follow-up. Neurosurgery 71:341-348, 2012

4. Dakwar E, Cardona RF, Smith DA, Uribe JS: Early outcomes and safety of the minimally invasive, lateral retroperitoneal transpsoas approach for adult degenerative scoliosis. Neurosurg Focus 28(3):E8, 2010

5. Glassman SD, Bridwell K, Dimar JR, Horton W, Berven S, Schwab F: The impact of positive sagittal balance in adult spinal deformity. Spine (Phila Pa 1976) 30:2024-2029, 2005

6. Glassman SD, Hamill CL, Bridwell KH, Schwab FJ, Dimar JR, Lowe TG: The impact of perioperative complications on clinical outcome in adult deformity surgery. Spine (Phila Pa 1976) 32:2764-2770, 2007

7. Hyun SJ, Rhim SC: Clinical outcomes and complications after pedicle subtraction osteotomy for fixed sagittal imbalance patients: a long-term follow-up data. J Korean Neurosurg Soc 47:95-101, 2010

8. Mummaneni PV, Tu TH, Ziewacz JE, Akinbo OC, Deviren V, Mundis GM: The role of minimally invasive techniques in the treatment of adult spinal deformity. Neurosurg Clin N Am 24:231-248, 2013

9. Schwab F, Dubey A, Gamez L, El Fegoun AB, Hwang K, Pagala M, et al: Adult scoliosis: prevalence, SF-36, and nutritional parameters in an elderly volunteer population. Spine (Phila Pa 1976) 30:1082-1085, 2005

10. Schwab F, Dubey A, Pagala M, Gamez L, Farcy JP: Adult scoliosis: a health assessment analysis by SF-36. Spine (Phila Pa 1976) 28:602-606, 2003

11. Schwab F, Farcy JP, Bridwell K, Berven S, Glassman S, 


\section{MISDEF algorithm}

Harrast J, et al: A clinical impact classification of scoliosis in the adult. Spine (Phila Pa 1976) 31:2109-2114, 2006

12. Schwab FJ, Hawkinson N, Lafage V, Smith JS, Hart R, Mundis $\mathrm{G}$, et al: Risk factors for major peri-operative complications in adult spinal deformity surgery: a multi-center review of 953 consecutive patients. Eur Spine J 21:2603-2610, 2012

13. Silva FE, Lenke LG: Adult degenerative scoliosis: evaluation and management. Neurosurg Focus 28(3):E1, 2010

14. Smith JS, Shaffrey CI, Berven S, Glassman S, Hamill C, Horton $\mathrm{W}$, et al: Improvement of back pain with operative and nonoperative treatment in adults with scoliosis. Neurosurgery 65:86-94, 2009

15. Smith JS, Shaffrey CI, Berven S, Glassman S, Hamill C, Horton W, et al: Operative versus nonoperative treatment of leg pain in adults with scoliosis: a retrospective review of a prospective multicenter database with two-year follow-up. Spine (Phila Pa 1976) 34:1693-1698, 2009

16. Tormenti MJ, Maserati MB, Bonfield CM, Okonkwo DO,
Kanter AS: Complications and radiographic correction in adult scoliosis following combined transpsoas extreme lateral interbody fusion and posterior pedicle screw instrumentation. Neurosurg Focus 28(3):E7, 2010

17. Wang MY, Mummaneni PV: Minimally invasive surgery for thoracolumbar spinal deformity: initial clinical experience with clinical and radiographic outcomes. Neurosurg Focus 28(3):E9, 2010

Manuscript submitted January 14, 2014.

Accepted March 25, 2014.

Please include this information when citing this paper: DOI: 10.3171/2014.3.FOCUS1413.

Address correspondence to: Kai-Ming Fu, M.D., Department of Neurosurgery, Weill Cornell Medical College, 525 E. 68th St., Box 99, New York, NY 10065. email: kaimingfu@gmail.com. 